\title{
Recent advances in radiation oncology
}

\author{
Yasushi Nagata
}

Received: 5 June 2014/ Accepted: 6 June 2014/ Published online: 26 June 2014

(C) Japan Society of Clinical Oncology 2014

Stereotactic radiotherapy (SRT) for extracranial tumors has been referred to as stereotactic body radiation therapy (SBRT) or stereotactic ablative radiotherapy (SABR) and has been used recently to treat primary lung cancer and liver cancer [1].

The advantage of SBRT, with a smaller irradiated volume enabled by more precise set-up, is hypofractionated radiotherapy leading to a shorter treatment course of a week. Its clinical significance in both inoperable and operable T1N0M0 primary lung cancer has been reported throughout the world. Its advances in physics and technology are marvelous. However, its biological basis is still controversial, especially regarding whether the linearquadratic (L-Q) model can be applied for this single highdose radiotherapy. In this issue, Dr. Song, who is one of the world's leading radiation biologists, has written a critical review article. In this paper, he looks at the importance of vascular damage caused by the effect of a single high dose on tumor cell death. A new biological model taking vascular damage into consideration may therefore be required for SBRT.

On the other hand, the advances in intensity-modulated radiotherapy (IMRT) throughout the world are remarkable. This is a new technique for achieving optimal dose distributions using a multi-leaf collimator and computer technology. In Japan, more than 130 institutions make clinical use of IMRT. The cancers treated by IMRT include prostate cancer, head and neck cancer, brain tumor and all other localized tumors. Clinical evidence demonstrating a decrease in toxicities and improvement in local control and survival has been emerging. Dr. Nakamura, who is one of the world's leading radiation oncologists, has written a critical review article from the clinical point of view. The topics are brain, head and neck, breast, lung, prostate, uterus, and spinal metastases. He was able to find several reports demonstrating decreases in toxicity, although he could not find evidence of improvements in local control and survival. The significance of IMRT on clinical outcome should be continuously evaluated.

Conflict of interest The author declares that he has no conflict of interest.

\section{Reference}

1. Nagata Y (2013) Stereotactic body radiotherapy (SBRT) for early stage lung cancer. Cancer Res Treat 45(3):155-161
Y. Nagata $(\bowtie)$

Department of Radiation Oncology, Graduate School of

Biomedical and Health Sciences, Hiroshima University,

Kasumi 1-2-3, Hiroshima 734-8551, Japan

e-mail: nagat@hiroshima-u.ac.jp 